\title{
Ne-(carboxymethyl)-lysine, White Matter, and Cognitive Function in Diabetes Patients
}

\author{
Jian-Hui Zhang, Hong-Zeng Xu, Qi-Feng Shen, Yong-Zhong Lin, Chang-Kai Sun, \\ Lin Sha, Yu-Song Ge, Ying Liu, Chun Wang
}

\begin{abstract}
Objective: To study the relationship of Ne-(carboxymethyl)-lysine level (CML) with microstructure changes of white matter (WM), and cognitive impairment in patients with type 2 diabetes mellitus (T2DM) and to discuss the potential mechanism underlying T2DM-associated cognitive impairment. Methods: The study was performed in T2DM patients $(\mathrm{n}=22)$ with disease course $\geq 5$ years and age ranging from 65 to 75 years old. A control group consisted of 25 sex- and age-matched healthy volunteers. Fractional anisotropy (FA) of several WM regions was analyzed by diffusion tensor imaging scan. Plasma CML levels were measured by enzymelinked immunosorbent assay, and cognitive function was assessed by Mini-Mental State Examination and Montreal cognitive assessment (MoCA). Results: The total Mini-Mental State Examination score in the patient group $(25.72 \pm 3.13)$ was significantly lower than the control group $(28.16 \pm 2.45)(\mathrm{p}<0.05)$. In addition, the total MoCA score in the patient group $(22.15 \pm 3.56)$ was significantly lower than the control group $25.63 \pm 4.12)(\mathrm{p}<0.01)$. In the patient group, FA values were significantly decreased in the corpus callosum, cingulate fasciculus, inferior fronto-occipital fasciculus, parietal WM, hippocampus, and temporal lobes relative to corresponding regions of healthy controls $(\mathrm{p}<0.05)$. Plasma CML level was negatively correlated with average FA values in the global brain $(\mathrm{r}=-0.58, \mathrm{p}<0.01)$ and MoCA scores $(\mathrm{r}=-0.47, p<0.05)$. Conclusions: In T2DM, WM microstructure changes occur in older patients, and elevations in CML may play a role in the development of cognitive impairment.
\end{abstract}

RÉSUMÉ: Ne-(carboxyméthyl)-lysine, substance blanche et fonction cognitive chez des patients diabétiques. Objectif: Le but de l'étude était d'examiner la relation entre le niveau de $\mathrm{N} \varepsilon$-(carboxyméthyl)-lysine (CML) et les changements dans la microstructure de la substance blanche (SB) et l'atteinte cognitive chez des diabétiques de type 2 (DT2), et de discuter des mécanismes possibles sous-jacents à l'atteinte cognitive associée au DT2. Méthode: L'étude a porté sur des patients atteints de DT2 $(\mathrm{n}=22)$ dont la maladie évoluait depuis 5 ans ou plus et qui étaient âgés de 65 à 75 ans. Le groupe témoin était constitué de 25 volontaires sains appariés pour le sexe et l'âge. L'anisotropie fractionnaire (AF) de plusieurs régions de la SB a été analysée par IRM du tenseur de diffusion. Les niveaux de CML ont été mesurés par dosage immuno-enzymatique et la fonction cognitive a été évaluée par le mini-examen de l'état mental et le Montreal cognitive assessment (MoCA). Résultats: Le score total au mini-examen de l'état mental du groupe de patients $(25,72 \pm 3,13)$ était significativement plus bas que celui du groupe témoin $(28,16 \pm 2,45)(\mathrm{p}<0,05)$. De plus, le score total au MoCA du groupe de patients $(22,15 \pm 3,56)$ était significativement plus bas que celui du groupe témoin $(25,63 \pm 4,12)(\mathrm{p}<0,01)$. Dans le groupe de patients, les valeurs d'AF étaient significativement diminuées dans le corps calleux, le faisceau cingulaire, le faisceau fronto-occipital inférieur, la SB pariétale, l'hippocampe et les lobes temporaux par rapport aux régions correspondantes chez les sujets témoins en bonne santé ( $\mathrm{p}<0,05)$. Le niveau de CML plasmatique était corrélé négativement aux valeurs moyennes d'AF dans le cerveau total $(\mathrm{r}=-0,58 ; \mathrm{p}<0,01)$ et les scores au MoCA $(\mathrm{r}=-0,47 ; \mathrm{p}<0,05)$. Conclusions: Dans le DT2, des changements se produisent dans la microstructure de la SB chez les patients plus âgés et l'augmentation de CML pourrait jouer un rôle dans l'apparition de troubles cognitifs.

Keywords: Type 2 diabetes mellitus, Magnetic resonance imaging, Diffusion tensor imaging, Ne-(Carboxymethyl) lysine, Cognitive impairment, Hippocampal atrophy

doi:10.1017/cjn.2015.398

Can J Neurol Sci. 2016; 43: 518-522

Patients with diabetes mellitus are at risk for developing mild to moderate cognitive impairment. ${ }^{1,2}$ The incidence of dementia, including Alzheimer's disease (AD) and vascular dementia (VD), is higher in individuals with diabetes mellitus than in those without. ${ }^{3,4}$ The mechanisms underlying this cognitive dysfunction are complex. In diabetic cognitive dysfunction patients, changes in cerebral perfusion, microstructure of white matter (WM), and increased cortical and especially hippocampal atrophy have been demonstrated. $^{5}$ Diffusion tensor imaging (DTI) is a tool for investigating the local properties of brain WM and detecting early abnormal microstructural changes, including demyelination and axonal injury. ${ }^{6}$ Hsu et $\mathrm{al}^{7}$ identified structural changes in
WM associated with cognitive dysfunction. Another important factor for the pathogenesis of chronic complications due to diabetes are the formation of advanced glycation end-products

From the Department of Neurology (JHZ, HZX, QFS, YZL, YSG, YL, CW), Second Hospital, Dalian Medical University, Dalian, China; Institute for Brain Disorder and

Disease, Dalian Medical University, Dalian, China (C-KS); Department of Radiology, Second Hospital, Dalian Medical University, Dalian, China (LS).

Received January 20, 2015. Final Revisions Submitted November 20, 2015. Correspondence to: Yong-Zhong Lin, Department of Neurology, Second Hospital, Dalian Medical University, Dalian 116027, China; or Chang-Kai Sun, Institute for Brain Disorder and Disease, Dalian Medical University, Dalian 116044, China. Email: lin19671024@163.com or cksun110@vip.sina.com. 
(AGEs), ${ }^{8}$ and they have been linked to both $\mathrm{VD}^{9}$ and $\mathrm{AD} .{ }^{10}$ $\mathrm{Ne}$-(Carboxymethyl)lysine (CML) is a predominant and well-studied form of $\mathrm{AGE}^{11}$ that is significantly elevated in the cerebrospinal fluid of patients suffering from $\mathrm{AD}$ and $\mathrm{VD}$ relative to controls. ${ }^{12}$ In the current study, we examined the most commonly used DTI metric, fractional anisotropy (FA), to determine if WM microstructure is altered in older communitydwelling adults with type 2 diabetes mellitus (T2DM). In addition, the relationship among plasma CML level, FA value, and cognitive function scores was investigated to further understand potential mechanisms underlying cognitive impairment T2DM patients.

\section{Materials and Methods}

\section{Participants}

The study was approved by the Medical Ethics Committee of the Second Affiliated Hospital of Dalian Medical University, and written informed consent was obtained from each participant before magnetic resonance imaging (MRI). For the patient group, subjects were selected from outpatients and inpatients of the Department of Neurology, Second Affiliated Hospital of Dalian Medical University, from March 2012 to March 2014. Patient ages ranged from 65 to 75 years with T2DM spanning at least 5 years. Patients were right hand-dominant and Chinese-speaking. There was no history of other glucose-lowering drugs besides biguanide and alpha glycosidase inhibitor. For the control group, subjects were recruited from patient families and the community. They were sex-, age-, and education-matched controls and Chinese-speaking.

Patients and controls were excluded based on the following criteria: (1) history of cardiovascular disease (e.g. hypertension, coronary heart disease, myocardial infarction), (2) history of central nervous system disease (e.g. transient ischemic attack or stroke, Parkinson's disease, severe brain injury, seizure, multiple sclerosis, infectious diseases of central nervous system), (3) standard contraindications to MRI, (4) hepatic dysfunction, (5) renal dysfunction, (6) a psychiatric or neurological disorder (unrelated to diabetes) that could influence cognitive functioning (such as $\mathrm{AD}$ and VD), (7) substance abuse (alcohol, drugs, or others), and (8) diabetic peripheral neuropathy. For all participants, HbAlc and blood glucose were measured in the overnight fasting state.

All participants underwent a detailed and normative cognitive neuropsychology assessment, including Montreal cognitive assessment (MoCA) ${ }^{13}$ Mini-Mental State Examination (MMSE) ${ }_{1}^{14}$ and clinical dementia rating. ${ }^{15}$ All tests were conducted in Mandarin by the same trained licensed neurologist with experience in a fixed order who was blind to the two groups.

\section{CML}

Following an overnight fast (12-14 hours), a 5-mL blood sample was collected from each patient, and the level of CML was measured using an enzyme-linked immunosorbent assay kit (Westang Bio-tech, Shanghai, China) for quantitative analysis according to the manufacturer's protocol, as described previously. ${ }^{16}$

\section{DTI Acquisition}

DTI was performed on a 3.0T MRI scanner (Siemens, Malvern, PA) using an eight-channel phased-array head coil. First, conventional sagittal T1-weightedand fluid-attenuated inversion recovery
(FLAIR) images were scanned for routine MRI screening. The parameters for T1-weighted imaging were as follows: repetition time/ echo time $(\mathrm{TR} / \mathrm{TE})=2550 / 3.6 \mathrm{~ms}, \mathrm{TI}=1100 \mathrm{~ms}$, flip angle $=7^{\circ}$, field of view $(\mathrm{FOV})=230 \times 180 \mathrm{~mm}$, and matrix size $=512 \times 512$. The parameters for FLAIR imaging were as follows: TR/TE $=10800$ / $120 \mathrm{~ms}, \mathrm{TI}=2700 \mathrm{~ms}$, section thickness $=5 \mathrm{~mm}, \mathrm{FOV}=230 \times 180$ $\mathrm{mm}$, matrix size $=512 \times 512$, and modulation of flip angle for refocusing pulses. A single refocusing spin-echo echoplanar imaging sequence was used to acquire DTI data. The sequence parameters were as follows: TR/TE $=9800 / 92 \mathrm{~ms} ; \mathrm{b}$ value: 0 and $1000 \mathrm{~s} / \mathrm{mm}^{2}$, respectively; $F O V=240 \mathrm{~mm} \times 240 \mathrm{~mm}$; matrix size $=256 \times 256$, Thirty-one isotropically distributed diffusion weighted directions with $\mathrm{b}=1000 \mathrm{~s} / \mathrm{mm}^{2}$ and one additional image without diffusion gradients $\left(b=0 \mathrm{~s} / \mathrm{mm}^{2}\right)$ were also acquired.

\section{Image Processing and Analysis}

DTI images were processed using the diffusion toolbox implemented in the functional MRI of the brain (FMRIB) software library 5.0 (http://fsl.fmrib.ox.ac.uk/fsl). First, each subject volume was affine registered to the first $b_{0}$ volume using the image registration tool FLIRT, and brain voxels of DTI data were extracted using the Brain Extraction Tool. ${ }^{16,17}$ Upon removal of nonbrain tissue, FA maps were calculated using the DTI-FIT tool.

A voxel wise statistical analysis of the FA values between different groups with the tract-based spatial statistics method was performed. ${ }^{18}$ First, images from all individuals were aligned into a $1 \times 1 \times 1 \mathrm{~mm}^{3}$ Montreal Neurological Institute152 space. Next, a mean FA image of all aligned FA images was calculated and thinned to create the mean FA skeleton, which represented the centers of all tracts common for the group. The skeleton's FA threshold was $\geq 0.20$ to ensure that areas of low FA and/or high inter-subject variability were excluded from the analyses. Each subject's aligned FA data were then projected onto the skeleton. Group statistical analysis was conducted only on voxels within the WM skeleton mask. For each subject, the mean FA of brain tissue was calculated by averaging the WM across voxels. Group differences of FA were evaluated with a permutation-based randomized test and inference by using the threshold-free cluster enhancement method implemented in FSL. The statistical threshold for all image analyses was set to a cluster $\mathrm{p}<0.05$.

\section{Data Analysis}

All data are expressed as means \pm standard deviation and were analyzed by SPSS 19.0 software (Armonk, NY). Comparisons between groups in demographics, clinical data, cognitive data, and WM FA value were performed using a two-tailed $t$-test. Pearson correlation coefficient was used to evaluate the relationship of FA of the WM with clinical parameters and plasma CML level; $\mathrm{p}<0.05$ was considered statistically significant.

\section{RESUlTS}

\section{Demographic and Clinical Data}

Demographic and clinical characteristics of the patient and control groups are shown in Table 1. There were no significant differences between groups in age, gender, and education. The patient group had higher levels of fasting glucose, HbAlc, and plasma CML than the control group $(\mathrm{p}<0.05)$. The MMSE scores 
Table 1: Demographic and clinical data of the patients group and control group $(\bar{x} \pm S)$

\begin{tabular}{l|c|c}
\hline & Control group $(\mathbf{n}=\mathbf{2 5})$ & Patient group $(\mathbf{n}=\mathbf{2 2})$ \\
\hline Age & $71.75 \pm 6.84$ & $72.32 \pm 7.05$ \\
\hline Gender (F/M) & $14 / 11$ & $12 / 10$ \\
\hline Schooling years (years) & $12.72 \pm 2.21$ & $13.28 \pm 3.14$ \\
\hline Course of T2DM (years) & 0 & $9.8 \pm 2.58$ \\
\hline Fasting glucose $(\mathrm{mmol} / \mathrm{L})$ & $5.1 \pm 0.8$ & $8.5 \pm 2.3^{*}$ \\
\hline HbA1c $(\%)$ & $5.62 \pm 0.42$ & $7.86 \pm 1.43^{*}$ \\
\hline Plasma CML $(\mathrm{ng} / \mathrm{mL})$ & $232.3 \pm 98.5$ & $436.7 \pm 134.6^{*}$ \\
\hline MMSE & $28.16 \pm 2.45$ & $25.72 \pm 3.13^{*}$ \\
\hline CDR & $(0,0)$ & $(0,1.0)$ \\
\hline MoCA & $25.63 \pm 4.12$ & $22.15 \pm 3.56^{\dagger}$ \\
\hline
\end{tabular}

CDR scores are described as (minimum value, maximum value).

*Compared with the controls $\mathrm{p}<0.05$.

${ }^{\dagger}$ Compared with the controls $\mathrm{p}<0.01$.

$\mathrm{CDR}=$ clinical dementia rating; $\mathrm{CML}=\mathrm{N} \varepsilon$-(carboxymethyl)-lysine;

MMSE $=$ Mini-Mental State Examination; MoCA = Montreal cognitive assessment; T2DM, type 2 diabetes mellitus.

$(\mathrm{p}<0.05)(25.72 \pm 3.13$ and $28.16 \pm 2.45)$ and MoCA scores $(\mathrm{p}<0.01) \quad(22.15 \pm 3.56$ and $25.63 \pm 4.12)$ were significantly different between patient and control groups.

\section{DTI Image Analysis Results}

All participants completed the MRI scan. No obvious abnormal findings were seen in conventional MRI structural images (T1-weighted, T2-weighted, and FLAIR). The Fazekas scale scores in the control and patient groups ranged from 0 to 1 and 0 to 2 , respectively. The tract-based spatial statistics whole-brain analysis results revealed that there were no significant differences in FA values between the left- and right-sided regions in either patient or control groups (Figure 1). Relative to controls, FA values in the patient group were reduced in multiple regions, including the hippocampus, parietal and temporal lobes, corpus callosum, and its association fibers (Table 2). FA values in regions of frontal lobe, occipital lobe, and internal capsule were not significantly different.

\section{The Correlation Between CML and FA and MoCA}

Pearson correlation coefficient showed that there was a significant negative correlation between plasma CML level and average FA values in whole-brain WM of T2DM patients $(\mathrm{r}=-0.58, \mathrm{p}<0.01)$ (Figure 2). In addition, there was a negative correlation between plasma CML level and MoCA scores $(\mathrm{r}=-0.47, \mathrm{p}<0.05$; Figure 3 ). There was no correlation between plasma CML level and MMSE scores.

\section{DISCUSSION}

We found that the level of plasma CML in T2DM patients was significantly higher than controls. In addition, CML level was negatively correlated with cognitive function score, supporting our hypothesis that CML plays an important regulatory role in the manifestation of diabetic cognitive impairment. Deutekoms et al found CML in brain astrocytes and vascular endothelial cells and that brain CML levels in diabetic patients and a diabetic animal model are much higher than controls. ${ }^{19}$ Other studies demonstrated that CML levels were correlated with the severity of diabetic complications. ${ }^{20,21}$ Diabetes mellitus patients have an increased risk for mild to moderate cognitive impairment, likely from vascular dysfunction. ${ }^{1,2}$ A series of studies have shown using arterial spin labeling that cerebral blood flow is significantly reduced in elderly T2DM patients. ${ }^{22} \mathrm{~A}$ meta-analysis and a series of studies suggested that microangiopathy and vasculopathy play an important role in diabetic cognitive impairment. Nonenzymatic glycation reaction plays an important role in vascular dysfunction. ${ }^{23,24} \mathrm{CML}$, one of the irreversible end-products of the nonenzymatic glycation reaction, is the main form of AGEs. The deposition of AGEs occurs during the normal aging process, and diabetes mellitus accelerates this process. ${ }^{25-27}$ Studies have shown that the interaction of AGEs and their specific receptor may accelerate aging of the brain. ${ }^{28}$

In this study, we found no obvious structural changes on conventional MRI (both T1- and T2-weighted MRI) in T2DM patients, but we found a significant decrease in FA value in T2DM patients in the hippocampus, corpus callosum, and its association fibers, including superior longitudinal fasciculus, cingulate fasciculus, and inferior fronto-occipital fasciculus. These findings identified destruction of the WM of elderly T2DM patients, especially in the WM regions associated with memory and recognition, but not in WM of the frontal lobe, occipital lobe, and internal capsule. Other studies have shown that T2DM patients often demonstrate cognitive impairment, such as processing

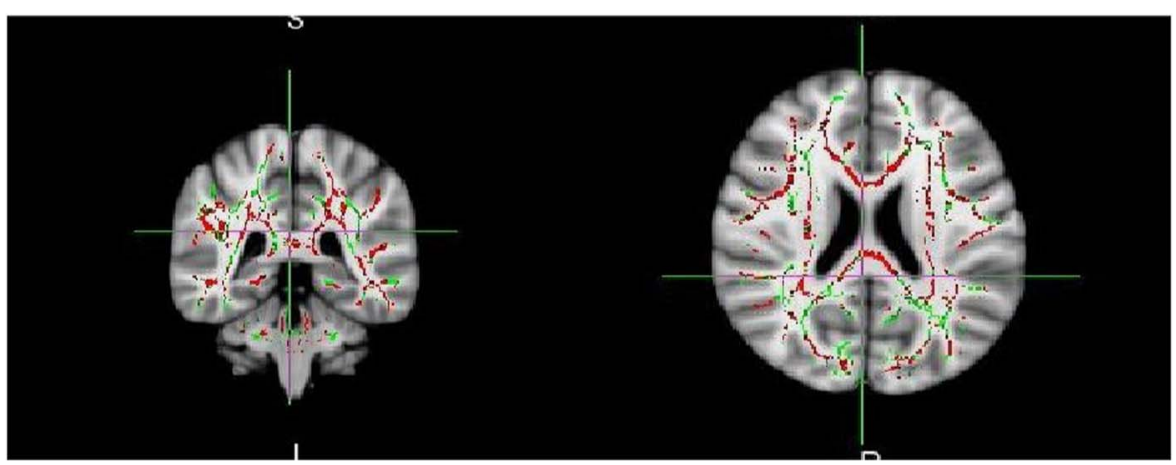

Figure 1: Tract-based spatial statistics whole-brain fractional anisotropy (FA) analysis. The regions of significantly reduced $F A$ values are red and the mean $F A$ skeleton is in green. 
Table 2: Comparison of FA values of brain regions between patient and control groups

\begin{tabular}{l|c|c|c}
\hline & Control group $(\mathbf{n}=\mathbf{2 5})$ & Patient groups $(\mathbf{n = 2 2})$ & p value \\
\hline Temporal lobe & $0.034 \pm 0.11$ & $0.031 \pm 0.08$ & $<.0 .001$ \\
\hline Hippocampus & $0.25 \pm 0.04$ & $0.32 \pm 0.11$ & $<0.001$ \\
\hline Parietal lobe & $0.35 \pm 0.08$ & $0.59 \pm 0.11$ & 0.011 \\
\hline Body of corpus callosum & $0.62 \pm 0.12$ & $0.61 \pm 0.07$ & 0.041 \\
\hline Splenium of corpus callosum & $0.65 \pm 0.08$ & $0.52 \pm 0.06$ & 0.028 \\
\hline Cingulate fasciculus & $0.63 \pm 0.05$ & $0.57 \pm 0.04$ & $<0.001$ \\
\hline Superior longitudinal fasciculus & $0.61 \pm 0.06$ & $0.51 \pm 0.07$ & 0.032 \\
\hline inferior fronto-occipital fasciculus & $0.58 \pm 0.08$ & $<0.001$ \\
\hline
\end{tabular}

information slowly, and it remains unclear whether the change is caused by WM microstructural abnormalities. ${ }^{29}$ Kodl et al identified microstructural WM abnormalities in a patient with long-standing type 1 diabetes patient, ${ }^{30}$ The FA values in the posterior corona radiate and the optic radiation were decreased, and these WM abnormalities were correlated with poor performance on some neurocognitive tests in the type 1 diabetes patient. ${ }^{30}$ Yau et al showed in middle-aged and elderly patients with T2DM in the absence of any obvious vascular pathology or psychiatric disorder that FA values in the frontal and temporal WM, which is associated with emotional and declarative memory, were significantly lower in T2DM patients. ${ }^{31}$ When DTI was used to investigate microstructural WM abnormalities among T2DM patients, these patients had significantly decreased FA value in bilateral frontal and total WM relative to controls. ${ }^{7,32}$ Meanwhile, there was a negative correlation between plasma CML level and average FA values in whole-brain WM in T2DM patients, suggesting that the accumulation of AGEs may be associated with microstructural WM changes.

Other factors also play an important role in diabetic cognitive impairment. Gold et al found hippocampal volume is negatively correlated with $\mathrm{HbA} 1$ in diabetes mellitus patients, ${ }^{33}$ prompting the importance of glucose control. The FA value in genu of corpus callosum was negatively correlated with disease course. ${ }^{30}$ Evidence

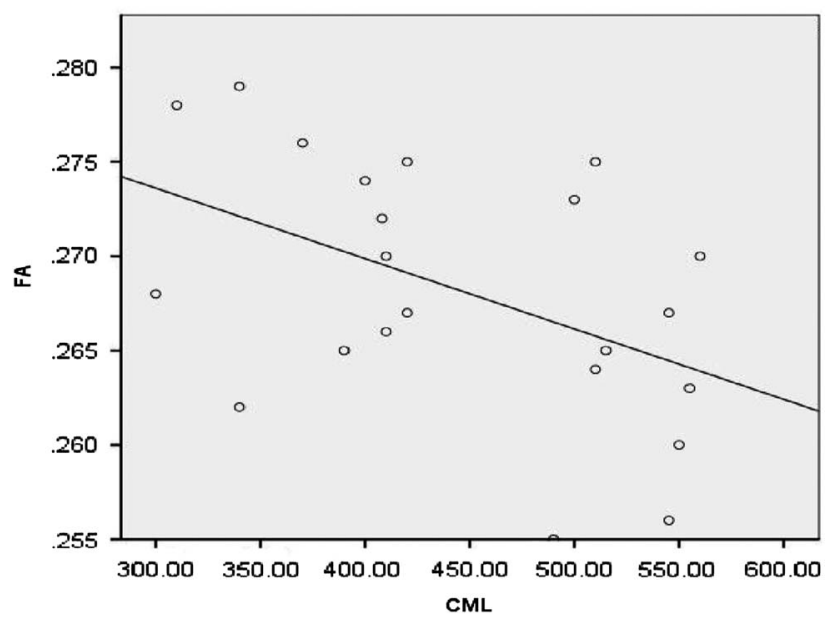

Figure 2: The correlation between plasma NE-(carboxymethyl)-lysine $(C M L)$ level and average fractional anisotropy (FA) values in global brain in the patient group. from DTI based on voxel analysis found that the longer the disease course of T2DM, the more serious the WM matter injury. ${ }^{7}$ However, this study did not determine the relationship between HbAlc and fasting blood-glucose with cerebral injury. In addition, other studies have found a relationship between disease course of T2DM peripheral neuropathy and autonomic neuropathy. ${ }^{34,35}$ Because the conclusions are contradictory, the mechanism underlying occurrence and development of diabetic cognitive impairment needs further study.

Our study had several limitations. First, the sample was restricted to old men, thereby limiting the generalizability of our results. In addition, the number of participants was relatively small, which may reduce the power of the statistical significance. Although this study discussed the relationship between the reduction of FA value and plasma CML level, the levels of CML in cerebrospinal fluid may be more relevant. ${ }^{12}$ Last, this study used only FA values to study the microstructure changes of brain white matter, and use of a multiple parameter comprehensive analysis may be more informative.

\section{CONCLUSIONS}

In summary, we have shown that there is a negative correlation between plasma CML and cognitive function score and that the FA value in WM of T2DM patients was significantly

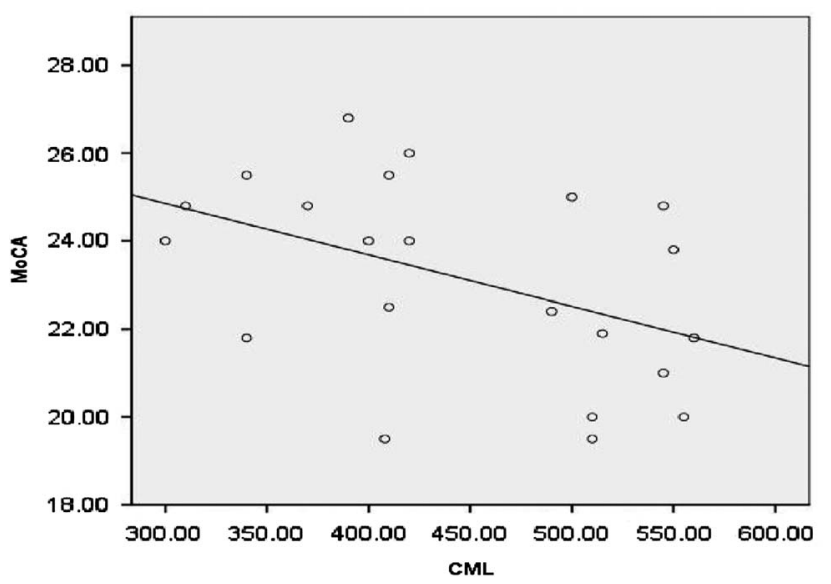

Figure 3: The correlation between plasma NE-(carboxymethyl)-lysine (CML) level and Montreal cognitive assessment (MoCA) scores in the patient group. 
decreased. These findings suggest that elevations in CML level and changes in WM microstructure in older T2DM patients may contribute to the development of cognitive impairment in these patients.

\section{ACKNOWLEDGMENTS}

The study was supported by the National Natural Science Foundation of China (no. 81571237), National Science and Technology Major Special Project on Major New Drug Innovation (No.2012ZX09503001-003), Natural Science Foundation of Liaoning Province of China (No. 2013023022), and Project of Liaoning Province Department of Education of China (No. L2014344).

\section{Disclosures}

The authors have nothing to disclose.

\section{REFERENCES}

1. McCrimmon RJ, Ryan CM, Frier BM. Diabetes and cognitive dysfunction. Lancet. 2012;379:2291-9.

2. Munshi M, Grande L, Hayes M, et al. Cognitive dysfunction is associated with poor diabetes control in older adults. Diabetes Care. 2006;29:1794-9.

3. Biessels GJ, Deary IJ, Ryan CM. Cognition and diabetes: a lifespan perspective. Lancet Neurol. 2008;7:184-90.

4. Biessels GJ, Staekenborg S, Brunner E, Brayne C, Scheltens P. Risk of dementia in diabetes mellitus: a systematic review. Lancet Neurol. 2006;5:64-74.

5. Ryan JP, Fine DF, Rosano C. Type 2 diabetes and cognitive impairment: contributions from neuroimaging. J Geriatr Psychiatry Neurol. 2014;27:47-55.

6. Bozzali M, Cherubini A. Diffusion tensor MRI to investigate dementias: a brief review. Magn Reson Imaging. 2007;25: 969-77.

7. Hsu JL, Chen YL, Leu JG, et al. Microstructural white matter abnormalities in type 2 diabetes mellitus: a diffusion tensor imaging study. Neuroimage. 2012;59:1098-105.

8. Brownlee M. Biochemistry and molecular cell biology of diabetic complications. Nature. 2001;414:813-20.

9. Southern L, Williams J, Esiri MM. Immunohistochemical study of $\mathrm{N}$-epsilon-carboxymethyl lysine (CML) in human brain: relation to vascular dementia. BMC Neurol. 2007;7:35.

10. Horie $\mathrm{K}$, Miyata $\mathrm{T}$, Yasuda $\mathrm{T}$, et al. Immunohistochemical localization of advanced glycation end products, pentosidine, and carboxymethyllysine in lipofuscin pigments of Alzheimer's disease and aged neurons. Biochem Biophys Res Commun. 1997;236:327-32.

11. Ikeda K, Higashi T, Sano H, et al. N (epsilon)-(carboxymethyl)lysine protein adduct is a major immunological epitope in proteins modified with advanced glycation end products of the Maillard reaction. Biochemistry. 1996;35:8075-83.

12. Bar KJ, Franke S, Wenda B, et al. Pentosidine and N(epsilon)(carboxymethyl)-lysine in Alzheimer's disease and vascular dementia. Neurobiol Aging. 2003;24:333-8.

13. Nasreddine ZS, Phillips NA, Bedirian V, et al. The Montreal Cognitive Assessment, MoCA: a brief screening tool for mild cognitive impairment. J Am Geriatr Soc. 2005;53:695-9.

14. Folstein MF, Folstein SE, McHugh PR. "Mini-mental state." A practical method for grading the cognitive state of patients for the clinician. J Psychiatr Res. 1975;12:189-98.

15. Morris JC. The Clinical Dementia Rating (CDR): current version and scoring rules. Neurology. 1993;43:2412-4.
16. Hu S, He W, Liu Z, Xu H, Ma G. The accumulation of the glycoxidation product $\mathrm{N}$ (epsilon)-carboxymethyllysine in cardiac tissues with age, diabetes mellitus and coronary heart disease. Tohoku J Exp Med. 2013;230:25-32.

17. Jenkinson M, Bannister P, Brady M, Smith S. Improved optimization for the robust and accurate linear registration and motion correction of brain images. Neuroimage. 2002;17:825-41.

18. Smith SM. Fast robust automated brain extraction. Hum Brain Mapp. 2002;17:143-55.

19. van Deutekom AW, Niessen HW, Schalkwijk CG, Heine RJ, Simsek S. Increased Nepsilon-(carboxymethyl)-lysine levels in cerebral blood vessels of diabetic patients and in a (streptozotocin-treated) rat model of diabetes mellitus. Eur J Endocrinol. 2008;158: 655-60.

20. Genuth S, Sun W, Cleary P, et al. Glycation and carboxymethyllysine levels in skin collagen predict the risk of future 10-year progression of diabetic retinopathy and nephropathy in the diabetes control and complications trial and epidemiology of diabetes interventions and complications participants with type 1 diabetes. Diabetes. 2005;54:3103-11.

21. McCance DR, Dyer DG, Dunn JA, et al. Maillard reaction products and their relation to complications in insulin-dependent diabetes mellitus. J Clin Invest. 1993;91:2470-8.

22. Smith SM, Jenkinson M, Johansen-Berg H, et al. Tract-based spatial statistics: voxelwise analysis of multi-subject diffusion data. Neuroimage. 2006;31:1487-505.

23. Last D, Alsop DC, Abduljalil AM, et al. Global and regional effects of type 2 diabetes on brain tissue volumes and cerebral vasoreactivity. Diabetes Care. 2007;30:1193-9.

24. van Harten B, de Leeuw FE, Weinstein HC, Scheltens P, Biessels GJ. Brain imaging in patients with diabetes: a systematic review. Diabetes Care. 2006;29:2539-48.

25. Brownlee M. Advanced protein glycosylation in diabetes and aging. Annu Rev Med. 1995;46:223-34.

26. Furth AJ. Glycated proteins in diabetes. $\mathrm{Br} \mathrm{J}$ Biomed Sci. 1997;54:192-200.

27. Yaffe K, Lindquist K, Schwartz AV, et al. Advanced glycation end product level, diabetes, and accelerated cognitive aging. Neurology. 2011;77:1351-6.

28. Takeuchi M, Yamagishi S. TAGE (toxic AGEs) hypothesis in various chronic diseases. Med Hypotheses. 2004;63:449-52.

29. Reijmer YD, Brundel M, de Bresser J, et al. Microstructural white matter abnormalities and cognitive functioning in type 2 diabetes: a diffusion tensor imaging study. Diabetes Care. 2013;36:137-44.

30. Kodl CT, Franc DT, Rao JP, et al. Diffusion tensor imaging identifies deficits in white matter microstructure in subjects with type 1 diabetes that correlate with reduced neurocognitive function. Diabetes. 2008;57:3083-9.

31. Yau PL, Javier D, Tsui W, et al. Emotional and neutral declarative memory impairments and associated white matter microstructural abnormalities in adults with type 2 diabetes. Psychiatry Res. 2009; 174:223-30.

32. Falvey CM, Rosano C, Simonsick EM, et al. Macro- and microstructural magnetic resonance imaging indices associated with diabetes among community-dwelling older adults. Diabetes Care. 2013;36:677-82.

33. Gold SM, Dziobek I, Sweat V, et al. Hippocampal damage and memory impairments as possible early brain complications of type 2 diabetes. Diabetologia. 2007;50:711-9.

34. Chen HT, Lin HD, Won JG, et al. Cardiovascular autonomic neuropathy, autonomic symptoms and diabetic complications in 674 type 2 diabetes. Diabetes Res Clin Pract. 2008;82: 282-90.

35. Hsu WC, Chiu YH, Chen WH, Chiu HC, Liou HH, Chen TH. Simplified electrodiagnostic criteria of diabetic polyneuropathy in field study (KCIS No. 14). Neuroepidemiology. 2007;28:50-5. 